As part of a proposed symposium on the importance of exposure assessment in epidemiology for hazard identification and risk characterization, this presentation will summarize progress to date in the adoption of stronger exposure assessment methodologies in epidemiological studies of shift work, and offer ideas for next steps. A particular focus will be placed on the refinement of measurement techniques to assess long-term exposure to shift work (e.g., roster-based assessment and validity studies), and on quantitative considerations of important co-exposures (e.g., light-at-night)

\section{Occupational Respiratory Disease}

\section{A.1 ASSESSING THE UNDER-ESTIMATION OF OCCUPATIONAL RESPIRATORY DISEASES IN TAIWAN: ANALYSES OF DISEASE BURDENS AND HEALTHCARE COSTS}

${ }^{1}$ Chung-Yen Chen*, ${ }^{2}$ Yawen Cheng. 'Taipei City Hospital, Renai Branch, Taipei City, Taiwan; ${ }^{2}$ Institute of Health Policy and Management, National Taiwan University, Taipei City, Taiwan

\subsection{6/OEM-2019-EPI.58}

Background Respiratory diseases, including pneumoconiosis, asthma, chronic obstructive pulmonary disease (COPD) and respiratory malignancies, are featured by their multi-etiological nature and long latency periods, adding to the difficulties in recognizing their work-relatedness. Due to their heavy overall disease burdens and high healthcare spending, examining the fraction of respiratory diseases attributable to occupational factors is helpful in understanding the magnitude of under-estimation of occupational injuries and diseases.

Methods Prevalence rates of various occupational exposures were assessed retrospectively with self-reported surveys or job-exposure matrices. Relative risks of theses exposures were drawn from international epidemiological literatures. The above two parameters were used to calculate population attributable risk percentages (PAR\%), and with that the numbers of visiting and amounts of payment attributable to occupational factors were estimated based on the claim data of the National Health Insurance (NHI). The estimates were compared with the data of actual payment by the Workers' Compensation Insurance (WCI).

Results We estimated that around 15000 patients visited medical facilities for occupational respiratory diseases in 2015, costing a total of 10 million USD. In contrast, less than 200 cases were approved by WCI in the same year. A 100 -fold gap between the estimated and actual payment was also noted. Estimation analyses further indicated that $9.6 \%$ of cases and $48.1 \%$ of healthcare costs were asbestosrelated.

Conclusion For occupational respiratory diseases with long latency periods and great causal complexity, the scope of under-estimation was substantial, and their medical expenses had been largely paid by NHI rather than WCI. To increase the visibility of occupational respiratory diseases, workplace exposure assessment and disease surveillance should be improved and public awareness of occupational diseases should be raised.

\section{A.3 DO AIRWAY INFLAMMATION AND AIRWAY RESPONSIVENESS MARKERS AT START OF APPRENTICESHIP PREDICT THEIR EVOLUTION DURING INITIAL TRAINING? A LONGITUDINAL STUDY}

${ }^{1}$ Valérie Demange*, ${ }^{2}$ Denis Zmirou-Navier, ${ }^{3}$ Abraham Bohadana, ${ }^{1}$ Pascal Wild. ${ }^{1}$ Institut National de Recherche et de Sécurité, Vandoeuvre-les-nancy, France; ${ }^{2}$ Ecole des Hautes Etudes de Santé Publique, Rennes, France; ${ }^{3}$ Pulmonary Institute, Jérusalem, Israël

\subsection{6/OEM-2019-EPI.59}

The natural history of airway inflammation and symptoms in occupations at risk of asthma is still not fully understood. We aimed to study the evolution during apprenticeship of inflammation markers, bronchial hyperresponsiveness (BHR) and symptoms in at-risk subgroups as defined from measurements of markers made shortly after the start of training.

Respiratory symptoms, $\mathrm{FEV}_{1}$ and airway resistance postbronchial challenge $(\mathrm{MBC})$ test results, fractional exhaled nitric oxide (FeNO) measurements, and eosinophils in nasal lavage fluid were longitudinally investigated in apprentice bakers, pastry-makers and hairdressers. Four baseline risk groups were defined, based on, (i) a high level of FeNO (NO), (ii) eosinophils $>1 \%$ (Eosino), (iii) $\mathrm{a} \geq 15 \%$ decrease in $\mathrm{FEV}_{1}$ during the $\mathrm{MBC}$ test (HR), and (iv) $\mathrm{a} \geq 50 \%$ increase in the resistance (Resist). The statistical analysis relied on mixed models.

At baseline, the inflammation markers were related to the MBC markers. There was no evidence to suggest that the baseline risk groups predict a differential evolution of the airway inflammation and bronchial responsiveness markers, or the asthma-like symptoms considered. The baseline risk groups defined from MBC test predicted the levels of MBC markers. Similarly, the baseline risk groups based on eosinophilic inflammation predicted the levels of markers for eosinophilia. Twelve possible new asthma cases were identified, only the HR risk group predicted their occurrence.

Among this young population, at-risk groups based on initial high levels of inflammation markers did not experience any worsening during the follow-up. Initial BHR predicted consistently high levels of all markers considered and occurrence of possible asthma. These results have been published in BMC Pulmonary Medicine (Demange et al. BMC Pulm Med. 2018 July 11;18(1):113).

\section{O3A.4 ABSTRACT WITHDRAWN}

\section{A.5 ENVIRONMENTAL DUST EXPOSURE FROM GOLD MINE WASTE DUMPS AND RESPIRATORY HEALTH EFFECTS IN JOHANNESBURG, SOUTH AFRICA}

\begin{abstract}
${ }^{1,2}$ Samantha Iyaloo* ${ }^{1,2}$ Tahira Kootbodien, ${ }^{1,2,3}$ Nisha Naicker, ${ }^{2,3,4}$ Angela Mathee, ${ }^{1,2}$ Spo Kgalamono, ${ }^{1,2}$ Kerry Wilson, 1,2 David Rees. 'National Institute for Occupational Health (NIOH), National Health Laboratory Services (NHLS), Johannesburg, South Africa; ${ }^{2}$ School of Public Health, University of the Witwatersrand, Johannesburg, South Africa; ${ }^{3}$ Environmental Health Department, Faculty of Health Sciences, University of Johannesburg, Johannesburg, South Africa; ${ }^{4}$ Environment and Health Research Unit, South African Medical Research Council, Johannesburg, South Africa
\end{abstract}

\subsection{6/OEM-2019-EPI.60}

Background Thousands of people living in close proximity to gold mine waste dumps (GMWDs), are exposed to wind- 